\title{
ACTITUD HACIA LA ESTADÍSTICA EN ESTUDIANTES DE PSICOLOGÍA: SISTEMATIZACIÓN DE UNA EXPERIENCIA FORMATIVA
}

\author{
Attitudes towards Statistics in Psychology Students: \\ Systematization of a Training Experience
}

Diego Palacios-Díaz ${ }^{1}$

diegopalacios@uti.edu.ec

\section{Estefanía Caisa-Lozada ${ }^{1}$}

estefaniacaisa@gmail.com

Michelle Camacho-Jumbo'

mcamachobelen97@gmail.com

${ }^{1}$ Universidad Tecnológica Indoamérica. Ecuador

Fecha de recepción: 5/4/2020

Fecha de aprobación: 15/7/2020

\section{Resumen}

El objetivo del presente artículo es reconstruir e interpretar críticamente una experiencia formativa en Estadística en estudiantes de Psicología desde el enfoque metodológico de la sistematización de experiencias (Jara, 2015). El punto de partida de esta sistematización consiste en abordar un problema específico del contexto: la actitud negativa hacia la estadística que manifiestan los estudiantes antes del inicio de un módulo de Enfoques Cuantitativos de Investigación en Ciencias Humanas. En el contexto iberoamericano, la investigación educativa contemporánea en el campo muestra continuamente dificultades actitudinales de los estudiantes hacia estas materias que potencialmente perjudican la calidad de las experiencias formativas. Por ello, en esta sistematización de experiencias detallamos los distintos momentos de un proceso formativo que buscó enfrentar los temores e indiferencia actitudinal de los estudiantes con miras a desarrollar óptimamente fundamentos metodológicos y procedimientos técnicos y prácticos para producir y analizar datos cuantitativos. Tras esta sistematización, las reflexiones del equipo investigador se orientan hacia la necesidad de desarrollar estrategias didácticas, relacionales y socioemocionales que respondan pertinentemente al problema actitudinal de los estudiantes hacia esta materia, así como también a pensar críticamente el lugar que, política y éticamente, ocupa y podría ocupar la estadística en el mundo contemporáneo.

Palabras clave: actitud, estadística, experiencia, sistematización.

\section{Abstract}

The aim of this article is to reconstruct and critically interpret a Statistics training experience in psychology students from the methodological approach of Systematization of Experiences (Jara, 2015). The starting point of this systematization is to tackle a context-specific problem: the negative attitude towards statistics that students manifest before starting a training module on Quantitative Research Approaches in Human Sciences. In the Ibero-American context, contemporary educational research in the field consistently shows students' attitudinal difficulties towards Statistics that potentially harm the quality of training experiences. From this Systematization of Experiences, we detail the different Stages of a training process that sought to face the fears and attitudinal indifference of the students with the aim of optimally developing methodological foundations and technical and practical procedures to produce and analyze quantitative data. After this systematization, the reflections of the research team are oriented towards the need to develop didactic, relational and socio-emotional strategies that respond appropriately to the student's attitude problem towards Statistics, as well as to think critically about the place that, politically and ethically, Statistics occupies and could occupy in contemporary world.

Keywords: attitude, experience, systematization, statistics. 


\section{Introducción}

La presente investigación reconstruye e interpreta críticamente una experiencia formativa en Estadísticas en estudiantes de Psicología desde los principios metodológicos de la sistematización de experiencias (Jara, 2015). En un contexto sociocultural, institucional y relacional que, con frecuencia, toma distancia del pensamiento estadístico y matemático, nos orientamos a profundizar en las disposiciones actitudinales que manifiestan los estudiantes antes de comenzar un módulo de formación en estos temas. El temor que en diversas oportunidades expresan los aprendices en materias de este campo del saber no representa un acontecimiento aleatorio, sino más bien es el efecto principal de cómo - en distintos contextos socioculturales y en distintas organizaciones - se promueve una enseñanza rígida, temible y despersonalizada sobre un saber que resulta fundamental para la comprensión y transformación de las sociedades contemporáneas.

Nuestro punto de partida para sistematizar esta experiencia es que necesitamos comprender ampliamente la actitud hacia la estadística de nuestros estudiantes. Ello no puede estar reducido a disposiciones cognitivas, afectivas y conductuales que explicarían individualmente por qué algunos estudiantes tienen éxito en estas materias y otros no. Tampoco puede estar reducido a la mera consideración de desarrollar estrategias didácticas neutrales que puedan (o no) modificar las actitudes de los estudiantes hacia los temas estadísticos. Por el contrario, sugerimos posicionar las experiencias formativas y las disposiciones de los estudiantes en un marco contextual más amplio que examine elementos socioculturales, institucionales, curriculares y relacionales que estructuran estos fenómenos, así como también evaluar cómo estos mismos pueden contribuir significativamente a construir experiencias formativas más enriquecedoras en el campo de la estadística.

Con ello en vista, hemos estructurado este artículo ofreciendo una revisión de las investigaciones contemporáneas desarrolladas en Iberoamérica sobre actitudes hacia la estadística, de modo tal que podamos situar nuestro problema a abordar. Posteriormente, ofrecemos los principios epistemológicos y metodológicos de la sistematización de experiencias, enfoque que ha guiado nuestra ruta investigativa. Luego, definimos algunos momentos e hitos centrales de la experiencia de formación en estadísticas con nuestros estudiantes $\mathrm{y}$, a partir de ello, reconstruimos e interpretamos críticamente los eventos vividos y compartidos. Finalmente, ofrecemos algunas recomendaciones didácticas, relacionales y socioemocionales que, pensamos, pueden contribuir a desarrollar mejores experiencias de enseñanza-aprendizaje de estos temas, así como también a valorar con mayor profundidad la relevancia de la estadística para la vida contemporánea.

\section{Revisión de la literatura}

En los últimos años, la actitud hacia la estadística se ha posicionado como un tema emergente en la investigación educativa desarrollada en Iberoamérica, especialmente en el contexto de la reformulación curricular que ha operado global y regionalmente en las universidades. Esto responde a que la estadística suele formar parte de distintos programas y planes de estudio de numerosas carreras universitarias. No obstante, las materias estadísticas suelen ser resistidas por los estudiantes, lo cual genera diversas dificultades disciplinares, didácticas y relacionales en el proceso formativo de los profesionales (Auzmendi, 1992; Estrada, 2002; Vilá \& Rubio, 2016).

Aun cuando no existe unanimidad respecto del término actitud en un sentido general (Estrada, 2002), puede ser considerado como una agrupación de tendencias inferidas que surgen en edades tempranas y que orientan las acciones individuales y colectivas de los sujetos (Armas, 2020). Además, aun cuando preliminarmente las actitudes hacia ciertos temas pueden ser favorables, las experiencias sociales y culturales que comparten los sujetos pueden influenciarlas $y$ hacer que evolucionen negativamente conforme avance el tiempo, como ocurre frecuentemente con la estadística (Estrada et al., 2013).

Particularmente, en lo relacionado con la actitud hacia la estadística, en las investigaciones clásicas sobre 
este tema existe un relativo consenso respecto de las dimensiones elementales de las actitudes (Auzmendi, 1992; Gil-Flores, 1999): una dimensión cognitiva que contempla elementos relacionados con el pensamiento y las creencias; una dimensión afectiva que incorpora elementos emocionales y sentimentales; y una dimensión conductual relacionada con acciones y tendencias a la acción hacia un tema determinado. Otros autores han desarrollado una visión cuatridimensional que integra elementos cognitivos, afectivos, valóricos y de dificultad percibida respecto de la Estadística como asignatura (Schau et al., 1995), los cuales han sido complejizados posteriormente incorporando dimensiones tales como competencia afectiva, valor, dificultad, interés y esfuerzo (Rodríguez-Santero \& Gil-Flores, 2019).

Para lo que es de interés en esta investigación, hemos catastrado algunas investigaciones iberoamericanas de los últimos cinco años que exploran las actitudes hacia la estadística en estudiantes universitarios. Para lo que es también de interés específico en nuestra investigación, hemos incorporado investigaciones realizadas en estudiantes de la carrera de Psicología, la cual configura nuestro propósito investigativo. En general, en este tema emergente existe una preocupación creciente por comprender las actitudes con las que los estudiantes enfrentan materias estadísticas y, del mismo modo, problematizar las estrategias didácticas y relacionales que los docentes utilizan (o no) para enseńar estos temas.

En este contexto, en un estudio realizado con estudiantes de la carrera de Orientación de San José (Costa Rica), se evidenció una actitud positiva hacia la estadística en el estudiantado $(N=223$; Edad 17-25 años; 64,2\% hombres, 35,8\% mujeres) tanto a nivel general como en dimensiones específicas del instrumento SATS (Survey of Attitudes Towards Statistics) de Schau et al. (1995), que incluye valoraciones sobre un componente afectivo, cognitivo, valor y dificultad hacia la estadística. En esta aproximación se encontraron diferencias estadísticamente significativas en la actitud percibida en función de las experiencias de formación previa en temas de estadística, de modo tal que aquellos estudiantes que habían recibido estadística en el marco de otro curso no específico de la materia $[M=82,2 ; D S=7,74]$ tuvieron una actitud más positiva que aquellos estudiantes que manifestaron no haber recibido estadística $[M=86,55 ; D S=7,29 ; t(217)=4,19, p=0,00]$ (García-Martínez et al., 2015). Desde este hallazgo, los autores enfatizan en la necesidad de implementar medidas curriculares que enlacen la estadística con otras materias del plan formativo, así como también incorporar cursos de Estadística Descriptiva dentro de dicho plan.

En la misma línea, en Bogotá (Colombia), un grupo de investigadores examinó las actitudes hacia la estadística de estudiantes universitarios $(N=223$; Edad; $M=22,1 ; D S=5,0 ; 79,8 \%$ mujeres, $20,2 \%$ hombres) pertenecientes a diversos programas de las escuelas profesionales de Ciencias Exactas e Ingeniería, Ciencias Económicas y Escuela Internacional de Gestión y Marketing (Pérez et al., 2015). Para ello utilizaron la AEE (escala de actitudes hacia la estadística) (Estrada, 2002), la AEC (escala de actitudes hacia la estadística (Cazorla et al., 1999) y una tercera escala que integra los puntajes de los instrumentos anteriores. Un hallazgo relevante de esta investigación es que los estudiantes, si bien reconocen la importancia que tiene la estadística para la vida cotidiana y profesional, tienen desconfianza con los usos posibles de esta disciplina y especialmente con la capacidad percibida y el gusto por desempeñarse en estos temas. Los autores enfatizan en la necesidad de generar ajustes curriculares y didácticos que involucren a los estudiantes de manera más positiva con estas importantes materias.

En Barcelona (España), Vilá y Rubio (2016) evaluaron las actitudes hacia la estadística de 203 estudiantes de Pedagogía (Edad $M=21$ años; 90\% mujeres, $10 \%$ hombres) que comenzaban una asignatura de Estadísticas aplicada a la educación, materia de carácter obligatorio en el plan de estudios correspondiente. Un hallazgo relevante de esta aproximación es que no solo describió la actitud inicial hacia la materia (niveles neutros-bajos), sino que también describió perfiles específicos para abordar los temas estadísticos, a partir de los datos obtenidos desde la escala de 
actitud hacia la estadística de Auzmendi (1992). Un primer perfil muestra una actitud desfavorable y con escasos niveles de ansiedad, conocimientos previos y habilidades numéricas; un segundo perfil evidencia una actitud favorable pero también ansiosa y preocupada; $y$ un tercer perfil tiene altos niveles de ansiedad. Respecto de estos perfiles, los autores son enfáticos en indicar la necesidad de considerar estas diferencias internas en los grupos e implementar estrategias didácticas que atiendan estas particularidades.

En la misma línea, Ordóñez et al. (2019) construyeron perfiles actitudinales hacia la estadística de 855 estudiantes universitarios del ámbito de la Educación en Madrid (España). Considerando elementos socioeducativos, autoconcepto académico y actitudes hacia la estadística en el ámbito académico presente y en el futuro profesional, los autores elaboraron cuatro grupos. Dos grupos preponderantes $(61,64 \%)$ se caracterizan por ser mujeres con actitudes desfavorables, alta ansiedad, bajo autoconcepto académico y que no consideran útil la estadística. Los otros grupos tienen actitudes favorables y tienen buen autoconcepto académico, tienen conocimientos estadísticos previos, consideran útiles estos temas y se diferencian en sus niveles de ansiedad hacia la materia. Los autores enfatizan nuevamente en la necesidad de generar estrategias pedagógicas que sean sensibles a estas diferencias individuales y grupales, abordando los componentes actitudinales que guían el accionar de los estudiantes en sus experiencias formativas en estadísticas.

También en España, un grupo de investigadores ha explorado las actitudes hacia la estadística en estudiantes de Psicología con una formación de base muy heterogénea (Comas et al., 2017). Mediante la escala de actitudes hacia la estadística de Estrada (2002) se estudiaron las actitudes de 108 estudiantes de los primeros tres cursos de titulación de la carrera, en una muestra compuesta fundamentalmente por mujeres. Algunos de los hallazgos relevantes de esta exploración es que los estudiantes presentan actitudes positivas hacia la estadística, aunque ligeramente superiores a la posición de indiferencia, y que, conforme avanzan los años en el plan formativo, los estudiantes tienden a manifestar actitudes más negativas hacia la estadística, lo cual se debe posiblemente a las estrategias didácticas utilizadas en la formación en estas materias.

Por su parte, Valencia (2017) describió las actitudes hacia la estadística en 106 estudiantes de Psicología (Edad 18-35 ańos) de una universidad ubicada en Lima (Perú). Utilizando la escala de actitudes hacia la estadística de Auzmendi (1992), el autor encontró predominantemente niveles negativos $(22,6 \%$ de los participantes) y muy negativos $(29,2 \%)$ en la actitud de los estudiantes hacia estos temas. Un hallazgo relevante de esta investigación es que aquellos estudiantes que han tenido la oportunidad de tener cursos de estadística presentan una actitud más positiva en cuanto a la utilidad que tiene esta materia para su vida académica y profesional que aquellos que no $[M$ $=19,27 ; D S=2,256 ; t(105)=-2,038, p=0,044]$. Por la misma razón, una de las sugerencias planteadas guarda relación con generar experiencias formativas que contemplen elementos didácticos y prácticos que faciliten una formación pertinente para los profesionales de la Psicología en esta materia.

En Tegucigalpa (Honduras), Landa (2015) identificó las actitudes hacia la estadística de 316 estudiantes de Psicología que participaron en una asignatura de Medición Psicológica. En esta aproximación se utilizó la escala de actitudes hacia la estadística de Auzmendi (1992), a partir de la cual se detectó que el $16 \%$ de los participantes tenía una actitud negativa hacia la estadística, mientras que el $43 \%$ manifestaba una actitud desfavorable. Un hallazgo relevante de esta investigación es que los estudiantes, si bien reconocen que la estadística es una materia altamente útil para la vida cotidiana y profesional $(M=3,52 ; D S=$ $0,816)$, tienden también a manifestarse indiferentes en cuanto a predisposición activa y positiva hacia la materia $(M=2,77 ; D S=0,90)$. En la línea de la reflexión educativa, otro hallazgo relevante de esta investigación es que la actitud hacia la estadística no varía en función del nivel formativo del estudiante, de modo tal que tiende a haber continuidad actitudinal entre quienes recién comienzan su formación y quienes están en los cursos más especializados. 
Desde esta panorámica iberoamericana que hemos presentado, podemos destacar algunas tendencias relevantes para lo que será nuestra experiencia investigativa. En primer lugar, que fundamentalmente se reportan actitudes tendientes a la indiferencia o a la negatividad hacia la estadística en distintos contextos y programas de estudio, lo cual confirma una tendencia recurrente respecto de las disposiciones actitudinales de estudiantes que comienzan o cursan asignaturas del campo de las estadísticas. En segundo lugar, que la evidencia investigativa respecto de las experiencias formativas previas es mixta, en tanto en algunos casos pueden potenciar una actitud positiva en los estudiantes, así como también en otros pueden manifestar actitudes más desfavorables conforme avancen los años y se sumen nuevas asignaturas, o bien, que las actitudes no varíen entre estudiantes que comienzan cursos de estadísticas y otros que ya están cursando asignaturas avanzadas en este campo. En tercer lugar, que es preciso considerar elementos idiosincráticos y socioeducativos que contribuyan a detectar perfiles actitudinales hacia temas y materias de estadística, puesto que los estudiantes tienden a agruparse en actitudes desfavorables con percepciones de inutilidad de las estadísticas, así como también en actitudes favorables con distintos niveles de ansiedad respecto a la capacidad percibida para afrontar con éxito las tareas y actividades propias de esta disciplina.

Pensamos que las consideraciones planteadas son relevantes para formular experiencias de aprendizaje que, de modo relevante y pertinente, permitan abordar elementos actitudinales hacia la estadística que orientan la acción individual y colectiva de estudiantes universitarios. Estos aportes empíricos han guiado las decisiones que hemos tomado en el proceso formativo que a continuación presentamos, y que consiste en la sistematización de experiencia de un módulo de estadísticas compartido con un grupo de estudiantes de Psicología de una universidad ecuatoriana.

\section{Marco metodológico}

Nuestra investigación se posiciona desdelos principios epistemológicos y metodológicos de la sistematización de experiencias (Jara, 2015). En este marco, la experiencia como unidad de análisis es comprendida como un proceso sociohistórico de carácter dinámico y complejo que involucra elementos personales y colectivos y que trasciende la mera consideración de simples hechos o acontecimientos puntuales. La experiencia, en tanto fenómeno humano, implica también una integración compleja de elementos objetivos y subjetivos de la realidad histórico-cultural en que nos desenvolvemos $y$, como tal, constituye una fuente enriquecida para la elaboración de saberes y prácticas que nos posibiliten comprender el mundo y transformarlo (Freire, 2012, 2014).

En su calidad de enfoque metodológico, la sistematización de experiencias (en adelante, SE) es aquella "interpretación crítica de una o varias experiencias que, a partir de su ordenamiento y reconstrucción, descubre o explicita la lógica y el sentido del proceso vivido en ellas" (Jara, 2015, p. 77). Como equipo de investigación hemos adherido a la SE porque es un enfoque pertinente para producir y generar saberes que nos posibiliten reconstruir e interpretar críticamente las experiencias que vivimos y, con ello, comprenderlas teórica y prácticamente en su complejidad y profundidad con miras a orientarlas como herramientas de transformación de las distintas dimensiones de la realidad social en que participamos.

Concretamente, en esta SE hemos apostado por sistematizar nuestra experiencia de participación en el curso de Metodología de la Investigación Científica impartido en el segundo semestre de 2019 para estudiantes de Psicología de una universidad de la zona Sierra Centro de Ecuador. Esto se realiza primariamente desde el reconocimiento de que toda experiencia se despliega a través de múltiples dimensiones contextuales integradas que ofrecen las condiciones de posibilidad, las situaciones y las circunstancias temporales y espaciales específicas que dan el matiz singular a cada una (Jara, 2015). Del mismo modo, toda experiencia es relacional, lo cual implica tanto una vinculación física, emocional e intelectual entre seres humanos como su participación en las relaciones de poder que configuran el orden y la realidad social, a partir de dinámicas complejamente 
interconectadas de dominación, subordinación, solidaridad y resistencia.

Desde estas consideraciones, la experiencia que sistematizamos puede comprenderse contextualmente desde tres dimensiones: contexto institucional, contexto microcurricular y contexto relacional de la sala de clases (Figura 1). En relación con la primera dimensión, el modelo educativo de la institución en la que se lleva a cabo esta SE está orientado desde el enfoque socioformativo, cuya principal apuesta consiste en desarrollar el talento de personas, organizaciones y comunidades por medio del abordaje y resolución de problemas del contexto, lo cual se lleva a cabo a través de proyectos colaborativos que buscan impactar directa o indirectamente en el mejoramiento de las condiciones de vida de las sociedades humanas (Tobón, 2017). El modelo socioformativo guía institucionalmente los procesos y prácticas de formación de profesionales y ejerce una importante influencia, especialmente, en la estructuración del currículo y en los perfiles de egreso de las carreras que imparte la universidad.

El contexto microcurricular de esta SE está configurado desde las directrices del plan formativo de la carrera de Psicología, cuya cátedra de Metodología de la Investigación Científica se ubica en el quinto nivel, lo cual da inicio al tercer año de formación profesional. El sílabo de este proyecto formativo define como competencia especifica la "aplicación, de manera responsable, de métodos y técnicas de investigación científica para desarrollar trabajos investigativos a través de procedimientos sistemáticos" (Palacios, 2020, p. 1). De la misma manera, esta asignatura contribuye a las competencias específicas del perfil de egreso de la carrera mediante la "aplicación de conocimientos matemáticos para solucionar problemas con base en el pensamiento analítico y actuando bajo las normas éticas de cada contexto" (Palacios, 2020, p. 1).
En este ámbito microcurricular, el mapa de formación de la cátedra de Metodología de la Investigación Científica indica que el problema general a ser abordado en el proyecto formativo es el "desarrollo de un proyecto de investigación rigurosamente fundamentado para abordar problemáticas psicosociales presentes en distintos ámbitos de la vida social" (Palacios, 2020, p. 1). Este mapa de formación se estructura a partir de cuatro talleres o unidades temáticas que se organizan secuencialmente de la siguiente manera: 1) Introducción a los Métodos de Investigación en Ciencias Humanas; 2) Enfoque Cuantitativo de Investigación en Ciencias Humanas; 3) Enfoque Cualitativo de Investigación en Ciencias Humanas; 4) Proyecto de Investigación.

Desde estos elementos microcurriculares emerge el contexto relacional del aula en que realizamos esta SE. La experiencia a sistematizar se organiza desde las relaciones que como equipo establecimos con 31 estudiantes de quinto nivel que se encuentran organizados en dos grupos paralelos (Paralelo A y Paralelo B). Todos participamos de los cuatro talleres que estructuran el mapa de formación, cada uno de los cuales se extiende cuatro semanas en un régimen de 3 horas cronológicas por sesión. A propósito de los antecedentes bibliográficos que presentamos y desde los propósitos de trabajo compartidos con otros docentes y estudiantes de la institución, definimos conjuntamente que era pertinente abordar un problema propio de nuestra formación como psicólogos: el complejo aprendizaje de los temas de estadística en ciencias humanas. Con ello en vista, definimos que el producto final que debía generar nuestra experiencia en esta asignatura era un proyecto de investigación sólidamente fundamentado que resolviese algún problema del contexto, objetivo enmarcado en la orientación socioformativa del currículo de la universidad. 


\section{Figura 1. Dimensiones del contexto de sistematización de experiencia}

\section{Dimensiones del Contexto de Sistematización de Experiencia}

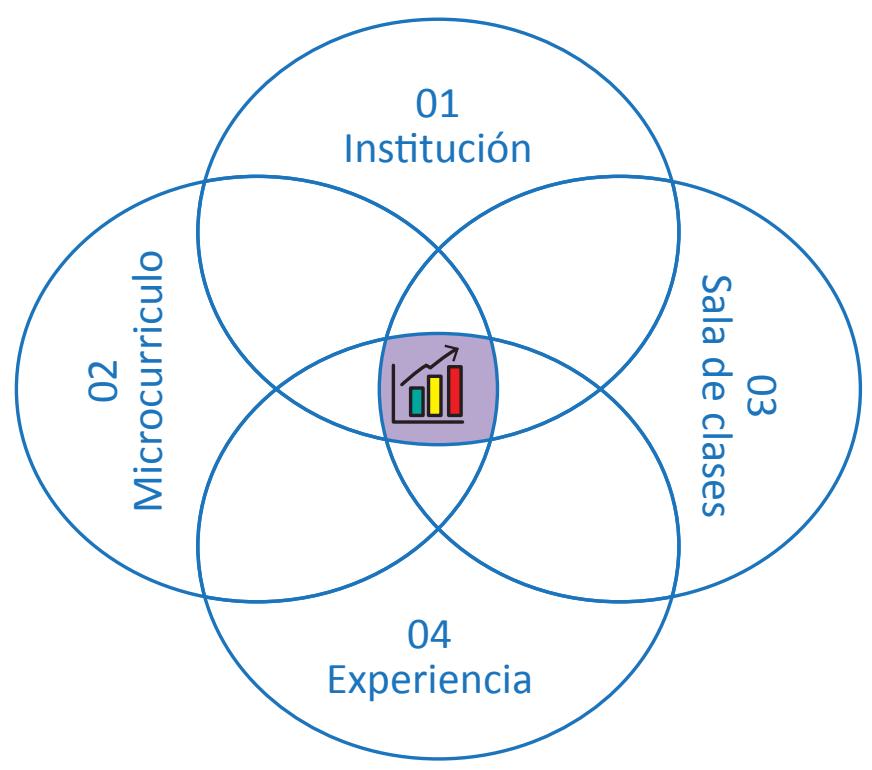

01 Contexto Institucional: Modelo Educativo Socioformativo 02 Contexto Microcurricular: Sílabo Metodología de la Investigación Científica 03 Contexto Relacional: Sala de Clases - Grupos de estudiantes que cursan MIC 04 Experiencia: Formación en Enfoques Cuantitativos de Investigación en Ciencias Humanas

Nota: Elaboración propia.

Siendo el propósito de esta experiencia formativa abordar las complejidades del aprendizaje de temas de estadística en ciencias humanas, definimos un doble propósito para afrontar específicamente las sesiones del Taller 2 del mapa formativo, denominado Enfoques Cuantitativos de Investigación en Ciencias Humanas (Figura 2). En principio, realizamos una simulación de un proceso de producción de datos cuantitativos, lo cual nos permitió entregar a los estudiantes recomendaciones técnicas y prácticas para producir datos de calidad, así como también ofrecer lineamientos para la construcción y gestión de bases de datos en el software IBM SPSS Statistics. Esta simulación también nos permitió realizar una breve caracterización socioeducativa de los estudiantes y una medida screening de la actitud hacia la estadística que presentaban antes de comenzar el taller correspondiente (Figura 3). Para este fin utilizamos la escala SATS-36 (Survey of Attitudes Toward Statistics) en su versión adaptada al español (Rodríguez-Santero \& Gil-Flores, 2019), la cual mide las actitudes que los estudiantes presentan hacia la estadística en cinco dimensiones: Competencia afectiva, Valor, Dificultad, Interés y Esfuerzo en valores que transitan desde 1 hasta 7 , en los que 1 es máxima actitud negativa y 7 es máxima actitud positiva. Esta medida screening estuvo acompañada, a su vez, de una lluvia de ideas que posibilitó verbalizar algunas de las principales dificultades y problemáticas percibidas por los estudiantes al momento de cursar asignaturas de estadística.

La subdimensión Competencia Afectiva evalúa la capacidad percibida para resolver problemas estadísticos; valor evalúa la utilidad y relevancia de las estadísticas en la vida personal y profesional; dificultad evalúa las actitudes sobre potenciales dificultades de los temas de estadísticas; interés evalúa las actitudes que manifiestan, justamente, interés o desinterés hacia estos temas; y esfuerzo evalúa las actitudes que los estudiantes están dispuestos a desplegar para cumplir con los requerimientos que les proponen materias de estadística (Rodríguez-Santero \& Gil-Flores, 2019). 
Figura 2. Mapa formativo - Taller 2

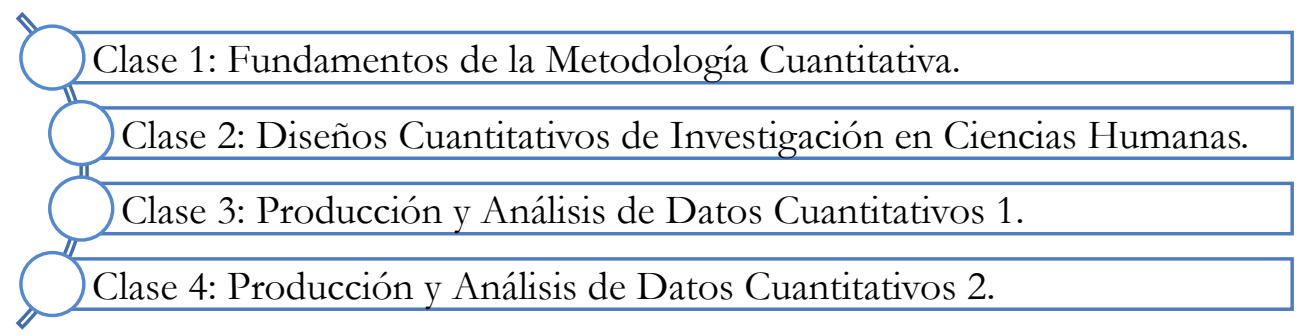

Nota: Elaboración propia.

Como se aprecia en la Figura 3, ambos paralelos comparten un perfil actitudinal similar al comienzo del taller de Enfoques Cuantitativos de Investigación en Ciencias Humanas, pues manifiestan una actitud negativa al evaluar la Dificultad de los temas estadísticos, así como también registran una actitud neutra en cuanto a su Competencia Afectiva, esto es, en la capacidad percibida que tendrán para afrontar con éxito aspectos problemáticos de la materia. Sin embargo, esta medida screening arrojó un elemento complementario importante ya que, a pesar de lo anterior y de no existir una actitud del todo positiva en cuanto a Interés, los estudiantes valoran positivamente la materia y también los esfuerzos que pueden realizar para cumplir con los propósitos del curso (actitud positiva en valor y esfuerzo).

Lo anterior se ve complementado con la información obtenida mediante una lluvia de ideas realizada con cada grupo, en la que registramos que su actitud negativa hacia la estadística se fundamenta principalmente en las experiencias previas que han vivido en distintos niveles de la educación formal. En principio, se han visto dificultados desde la enseñanza primaria con los temas de matemáticas y estadísticas y, además, ven en sus formadores en estas disciplinas a educadores estrictos y que explican con poca claridad y simpleza sus contenidos. A pesar de que durante sus dos primeros años de formación profesional han cursado tres materias relacionadas con matemáticas y estadísticas, los estudiantes manifiestan desconocimiento de cuál es la utilidad real que tienen para sus vidas cotidianas y profesionales, aun cuando intuitivamente reconocen su valor. Un elemento que distingue a un paralelo de otro es que el Paralelo A hace explícito su escaso interés por aprender estadísticas, mientras que el Paralelo B reconoce interés, pero manifiesta no saber cómo comenzar seriamente esta tarea. 
Figura 3. Caracterización socioeducativa y actitudinal de los estudiantes

Paralelo A

Género

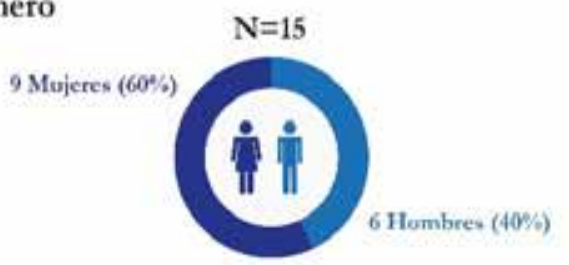

Edad

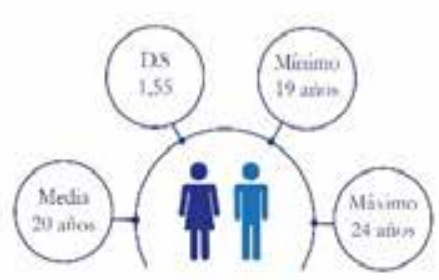

Actitud hacia la estadística

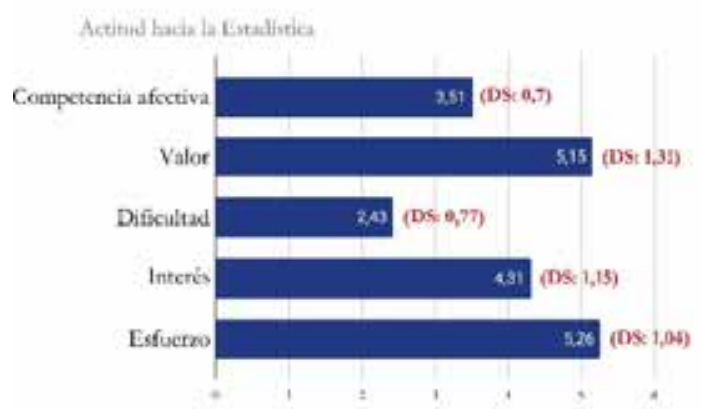

Principales dificultades y problemáticas de aprendizaje en Estadística

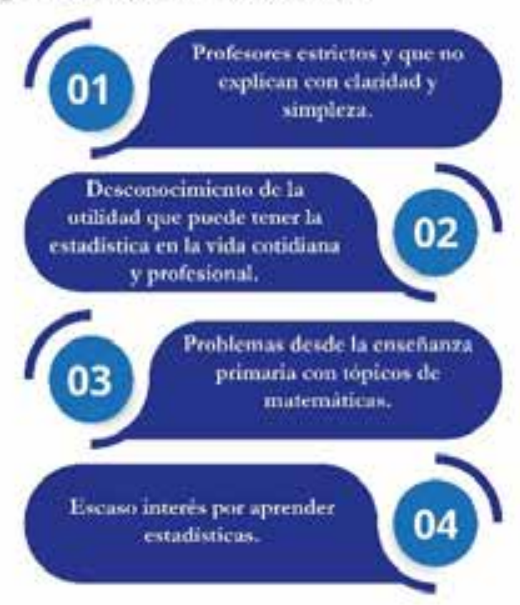

Materias cursadas en Ed. Superior en tópicos de Matemáticas y Estadisticas
Paralelo B

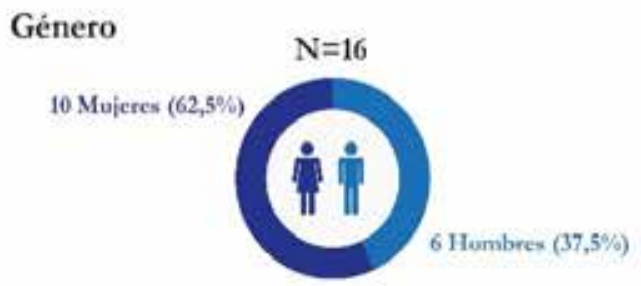

Edad

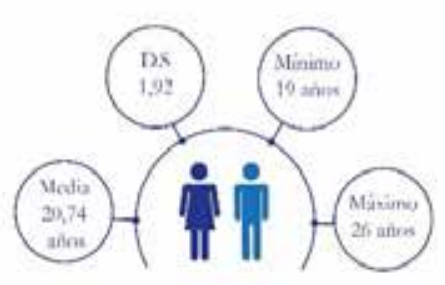

Actitud hacia la estadística

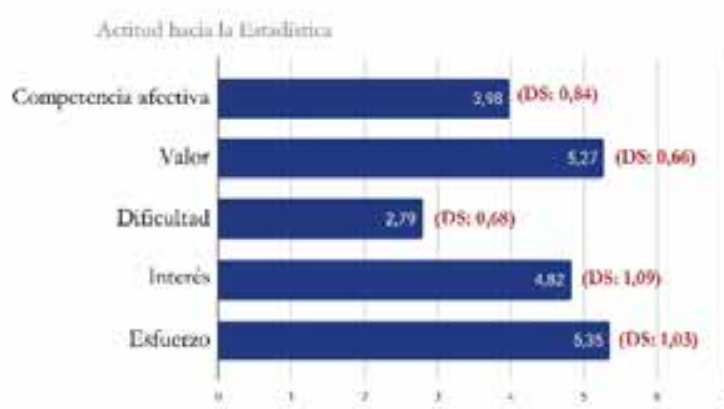

Principales dificultades y problemáticas de aprendizaje en Estadística

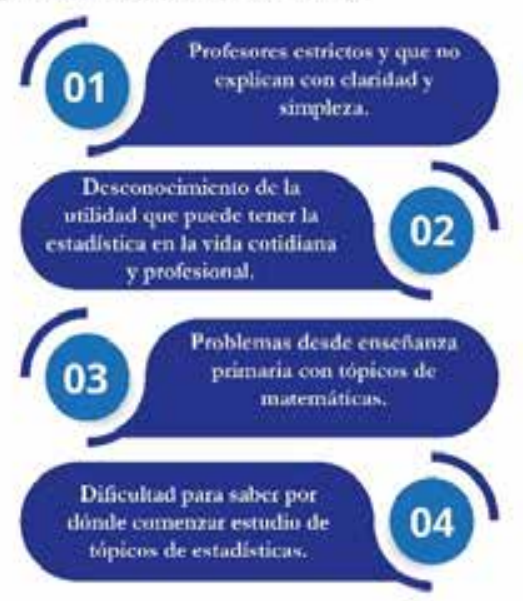

Materias cursadas en Ed. Superior en tópicos de Matemáticas y Estadísticas

Nota: Elaboración propia. 
Además, hemos utilizado distintas fuentes de información para reconstruir e interpretar críticamente nuestra experiencia (Figura 4), dentro de las cuales se destacan las dimensiones contextuales ya reportadas en nuestro marco metodológico, además de una bitácora de registro que el equipo docente utilizaba para identificar elementos propios de la dinámica de cada sesión y los productos que elaboraron los estudiantes para cumplir con los requisitos académicos: un informe de análisis estadístico y una cápsula audiovisual para socializar sus aprendizajes a la comunidad universitaria. Se suman las entrevistas semiestructuradas realizadas a los estudiantes tras haber finalizado el módulo de formación.

\section{Figura 4. Fuentes de información para la SE}

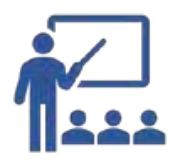

Bitácora de Registro de Clases

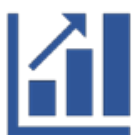

Informe de Análisis Estadístico
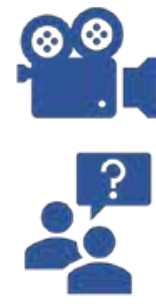

Cápsula Audiovisual de Socialización

Entrevista Semiestructurada

Nota: Elaboración propia.

\section{Resultados}

Presentamos a continuación los principales momentos de nuestra experiencia formativa en el Taller de Enfoques Cuantitativos de Investigación en Ciencias Humanas. El hilo conductor que hemos brindado a esta SE está conformado por tres momentos integrados: 1) Perdiendo el miedo; 2) El Oráculo; 3) Socializando nuestros aprendizajes, cada uno de los cuales nos permite abordar nuestro objetivo de reconstruir e interpretar críticamente la experiencia formativa de nuestro interés.

\section{Perdiendo el miedo}

Hemos titulado Perdiendo el miedo al primer momento de nuestra SE. Como mencionamos previamente, en la primera sesión del Taller 2 de la asignatura, aplicamos a cada uno de los paralelos la escala SATS-36, actividad que dio inicio a este módulo formativo. La aplicación a cada uno de los grupos duró alrededor de 20 minutos. Tras dicho momento, desarrollamos una lluvia de ideas para recoger opiniones sobre qué les parecía a los estudiantes comenzar un módulo de estadística. Transversalmente, las declaraciones de los estudiantes denotaban miedo, en el sentido amplio de la palabra. En uno de los grupos, una estudiante mencionó que "nuestro karma vuelve a aparecer" [bitácora de registro de clases]. Sobre la figura del karma se conversó respecto a algunos aspectos problemáticos de experiencias anteriores que los estudiantes habían tenido con las matemáticas y las estadísticas: el arquetipo del docente como una persona estricta y poco clara para explicar, la falta de claridad respecto de las posibles utilidades que pueden tener estos temas para la vida cotidiana y profesional y, especialmente, la apelación a que estos problemas han sido recurrentes a lo largo de sus trayectorias estudiantiles. La referencia al karma expresaba metafóricamente la dificultad para escapar de un tema complejo, así como también su inevitable reaparición en el contexto de sus experiencias formativas, esta vez de educación superior. De hecho, tras la participación en el módulo, los estudiantes en sus cápsulas audiovisuales expresaron con contundencia el miedo con el cual han significado previamente sus experiencias formativas en estas materias (Figura 5). 
Figura 5. Expresiones de miedo hacia la estadística en cápsulas audiovisuales
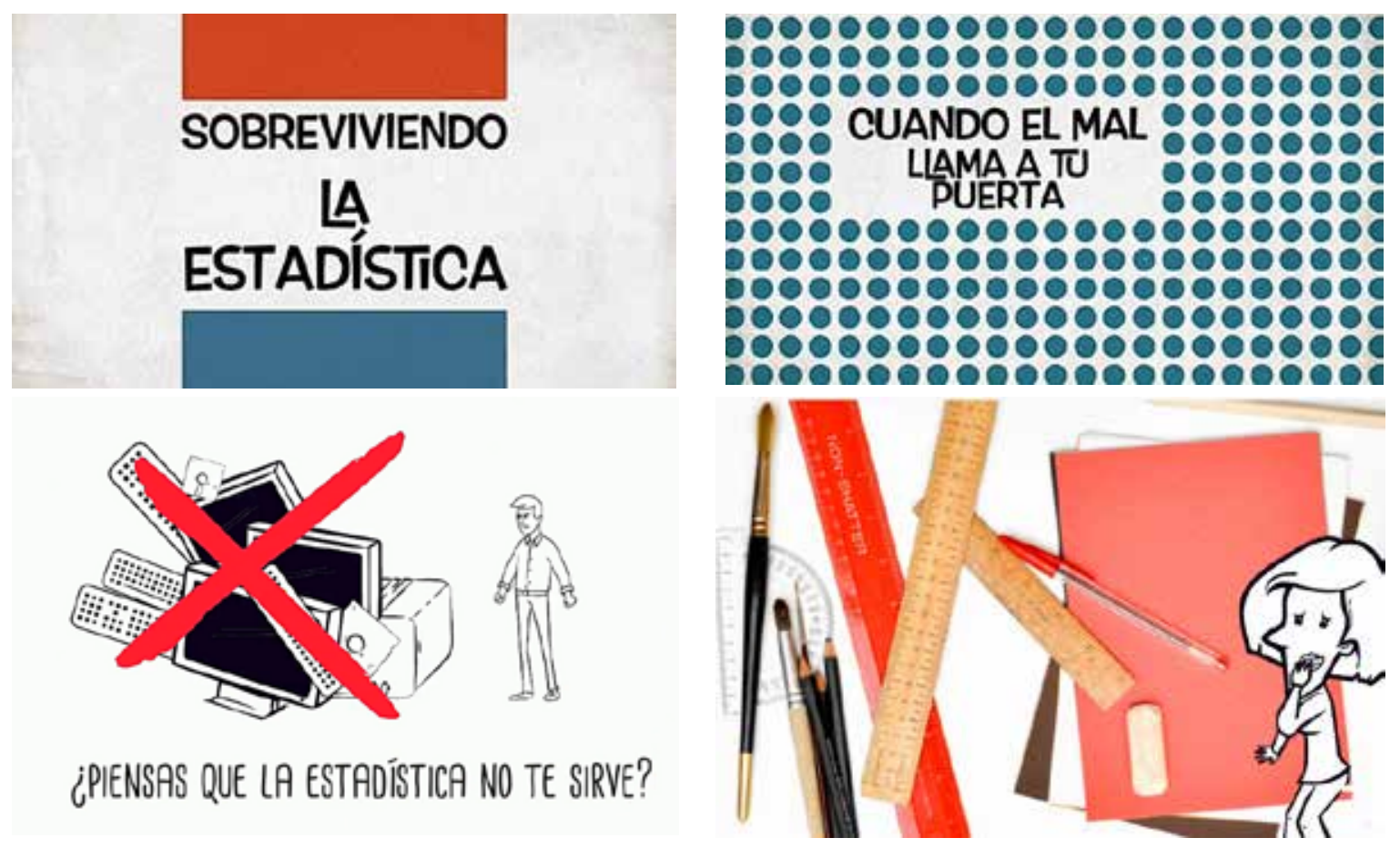

Nota: Extraídos de las cápsulas audiovisuales elaboradas por los estudiantes participantes.

El miedo fue un elemento simbólico que fue necesario atender en esta experiencia formativa. Habitualmente, nuestra formación en estadística está teñida por esta emoción básica, lo cual genera, en principio, evitación hacia los temas independientemente del grado de dificultad con que sean abordados y, de forma más profunda, nos descompromete actitudinal e intelectualmente de los desafíos que se nos presentan. Debido al screening realizado, consideramos como equipo docente que las sesiones del módulo debían orientarse desde el reconocimiento de esta emoción, lo cual nos invitó a pensar en estrategias didácticas que abordaran la naturaleza compleja y abstracta de los temas a trabajar. Esto fue especialmente necesario para la Sesión 1. Fundamentos de Metodología Cuantitativa y para la Sesión 2. Diseños Cuantitativos de Investigación en Ciencias Humanas. Por el carácter teórico-conceptual de estas dos primeras sesiones del módulo, buscamos plantear en los términos más simples posibles los fundamentos epistemológicos y metodológicos de los métodos cuantitativos, condición indispensable para que la estadística fuese presentada a los estudiantes como una herramienta útil y un lenguaje específico.

Para abordar el desafío anterior, definimos, por ejemplo, que el principal propósito de los métodos cuantitativos era generar las mejores fotografías posibles de determinados grupos o poblaciones. La noción de fotografía, trabajada conceptual y técnicamente como demoscopía social, la orientamos hacia comprender aspectos idiosincráticos y socioculturales de la población. Esto lo realizamos a través de preguntas sencillas, estrechamente ligadas con sus cotidianeidades. Por ejemplo, planteábamos preguntas como las siguientes: ¿Cuál es la probabilidad de ser diagnosticado con déficit atencional con hiperactividad si eres niño o niña? ¿Cómo crece o decrece esta probabilidad si consideramos el nivel socioeconómico al que pertenece tu familia? ¿Cómo crece esta probabilidad si consideramos tu origen étnico, al tratarse de un pais plurinacional?, entre otras.

Junto con lo anterior, definimos que los diseños de investigación son cajas de herramientas que nos 
permiten tomar decisiones ajustadas con nuestros propósitos o, en un lenguaje más sencillo, promovimos que diseñar podía ser definido como el arte de tomar buenas decisiones. También para facilitar esta comprensión, generamos un principio con ambos grupos: "Mientras mejores decisiones tomes, más alto es tu poder explicativo". Esto fue especialmente útil para enseñar conceptualmente a los estudiantes a comprender distintos tipos de diseños cuantitativos (experimentales, cuasiexperimentales y no experimentales), así como también distintos alcances de explicación (exploratorio-descriptivo-relacional-explicativo). Este arte de tomar decisiones debía responder a algunas preguntas elementales que nos sirvieron para trabajar conceptualmente algunos ejes claves del módulo: ¿quiénes participarán en nuestras investigaciones y en qué condiciones? (enfoques de muestreo), ¿qué técnicas utilizaremos y en qué condiciones? (producción de datos cuantitativos), y ¿qué haremos con los datos?, ¿cómo los leeremos? (análisis de datos en IBM SPSS Statistics).

Estos elementos fueron plasmados posteriormente en una actividad de construcción colectiva que nos permitió abordar el problema del contexto que habíamos definido: nuestra propia actitud hacia la estadística. Como ya contábamos con los datos del screening realizado, en la Sesión 2 definimos colectivamente qué haríamos con ellos. Para tomar una buena decisión, planteamos una metáfora conceptual que orientó la decisión grupal. De este modo, preguntamos a los participantes quién estaría en condiciones de correr una maratón en el curso de esta semana, es decir, ¿quiénes son capaces de correr 42 kilómetros? Como no había en nuestros grupos deportistas de alto rendimiento, redefinimos nuestra pregunta: ¿quiénes podrian participar en media maratón (21 kilómetros)? Uno de los 31 participantes dijo estar en condiciones. Volvimos a plantear nuestra pregunta: iquiénes podrían correr 10 kilómetros durante esta semana? Un tercio de los participantes se manifestó motivado y preparado para dicha tarea. Luego vino nuestra contra-pregunta para sensibilizar sobre la relevancia de tomar decisiones ajustadas para abordar nuestros datos: ¿por qué habriamos de correr maratones si no estamos preparados para correr siquiera un par de kilómetros? Esta pregunta orientó, en principio, la definición del alcance explicativo con el que trataríamos los datos que habíamos producido y, luego, nos permitió definir objetivos de investigación.

Para cumplir con lo anterior, presentamos a los estudiantes una matriz que señalaba que los objetivos de investigación que debían cumplir tenían dos condiciones (Figura 6): 1) ser operaciones cognitivas que 2) puedan ser traducidas a productos escritos (informes de investigación). Solo definiendo claramente aquello podíamos construir objetivos de investigación atendiendo a la siguiente secuencia: 1) enunciar una operación cognitiva que refleje el alcance de investigación definido; 2) enunciar nuestras variables y unidades de análisis; 3) indicar los sujetos participantes; y 4) el contexto en que ellos participan. La maratón que decidimos correr con cada grupo tuvo un alcance relacional, dado que nos propusimos describir profundamente tanto nuestras características socioeducativas (edad, sexo, paralelo) como nuestra actitud hacia la estadística (subdimensiones de SATS-36) y, si nuestra energía así lo permitía, examinaríamos algunas relaciones entre las variables (Figura 7). En un sentido general, definir objetivos de investigación nos permitió que los estudiantes lograsen comprender conceptualmente las formas en que nos aproximaríamos a los datos, y esto, a su vez, nos permitió disipar los temores iniciales que habían manifestado. Definir objetivos nos permitió también construir las preguntas necesarias que realizaríamos en la Sesión 3 a un nuevo participante en el curso: el Oráculo. 
Figura 6. Matriz para construcción de objetivos de investigación

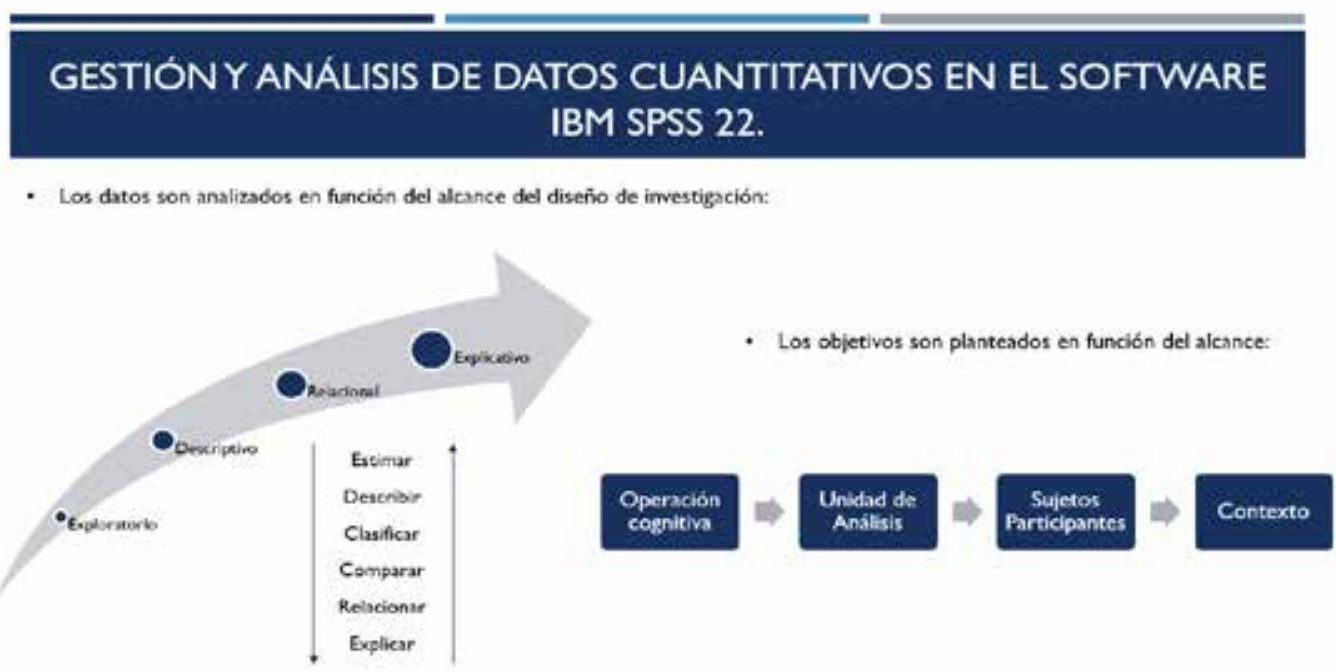

Nota: Extraído de las bitácoras de registro del equipo docente.

Figura 7. Objetivos de investigación construidos por los participantes

\section{GESTIÓNY ANÁLISIS DE DATOS CUANTITATIVOS EN EL SOFTWARE IBM SPSS 22.}

- Recomendación Primera: Definir bien nuestro enfoque y alcance $\rightarrow$ Nuestra investigación esta fundamentada en la Metodologia Cuantitativa (por qué). Su diseño es de tipo No Experimental (por qué) y su alcance es Relacional (por qué).

- Recomendación Segunda: Seguir nuestra matriz de construceión de objetivos. Un objetivo general : 3 objetivos especificos. OBJETIVO GENERAL

Relacionar los factores socioeducatimos (género, edad, paralelo) con la actitud hacia la estadistica en estudiantes de quinto semestre de psicologia, periodo B19, de la Universidad X. Ecuador.

OBJETIVOS ESPECIFICOS

Deseribir los factores socioeducativos (genero, edad, paralelo) de estudiantes de quinto semestre de psicologia, periodo B।9, de la Universidad X. Ecuador

Describir las actitudes hacia la estadistica de estudiantes de quinto semestre de psicologia, periodo BI9, de la Universidad X, Eeuador.

Estimar el grado de asociación entre factores socioeducativos y actitudes hacia la estadistica de estudiantes de quinto semestre de psicologia, período B19, de la Universidad X, Eccuador.

Nota: Extraído de las bitácoras de registro del equipo docente.

\section{El Oráculo}

Hemos titulado El Oráculo al segundo momento de nuestra SE. Tras el trabajo realizado en las Sesiones 1 y 2 del Taller 2 de la asignatura, orientamos el mapa de formación hacia la producción y análisis de datos cuantitativos. Como nuestra principal aspiración era involucrar afectiva y cognitivamente a los estudiantes en los temas de la materia, generamos en la Sesión 3 una actividad colectiva de construcción de un oráculo. Apelamos a esta metáfora conceptual para transmitir un principio básico de la gestión y análisis de bases de datos cuantitativos, y particularmente del software IBM SPSS Statistics: las fuentes 
de información pueden ser infinitas y, por la misma razón, conviene hacer las preguntas correctas. Con este principio, orientamos la estructuración de una base de datos en el software comentado, entregando algunas recomendaciones fundamentales para construir una adecuada fuente de información. Por ello hicimos hincapié en la correcta tabulación numérica de las variables trabajadas, en la definición precisa de los tipos de variables y sus correspondientes niveles de medición, en las especificaciones necesarias para registrar eventuales datos perdidos y en el ingreso preciso de etiquetas y valores para cada variable. Finalmente, el Oráculo fue bautizado como Quinto $A B$. sav y se constituyó como el participante que podría resolver algunas de las inquietudes que tenían los estudiantes respecto a sus idiosincráticas formas de abordar las problemáticas en estadísticas.

La participación colectiva de los grupos en la construcción de QuintoAB.sav generó un efecto positivo de apropiación de la experiencia de aprendizaje por parte de los estudiantes. Pensamos que esto se debió, en principio, a que comprendieron la lógica interna y el lenguaje con que trabajaba el software IBM SPSS Statistics en particular, así como también creemos que, posteriormente, este efecto se potenció porque aumentó su curiosidad respecto de verse reflejados en las respuestas que el Oráculo les podía ofrecer sobre sí mismos en sus distintas dimensiones, por ejemplo, de género, etáreas o socioeducativas. Al decir de una estudiante entrevistada tras la experiencia en el módulo: "El Oráculo nos permitió entendernos a nosotros mismos en nuestro problema de que no nos gusta la estadística, y también nos convenció de lo importante que es este conocimiento para nuestra profesión" [Carla, Paralelo B, Entrevista Semiestructurada]. Esto también se vio reflejado simbólicamente en las cápsulas audiovisuales desarrolladas por los estudiantes (Figura 9).

\section{Figura 8. El Oráculo QuintoAB.sav}

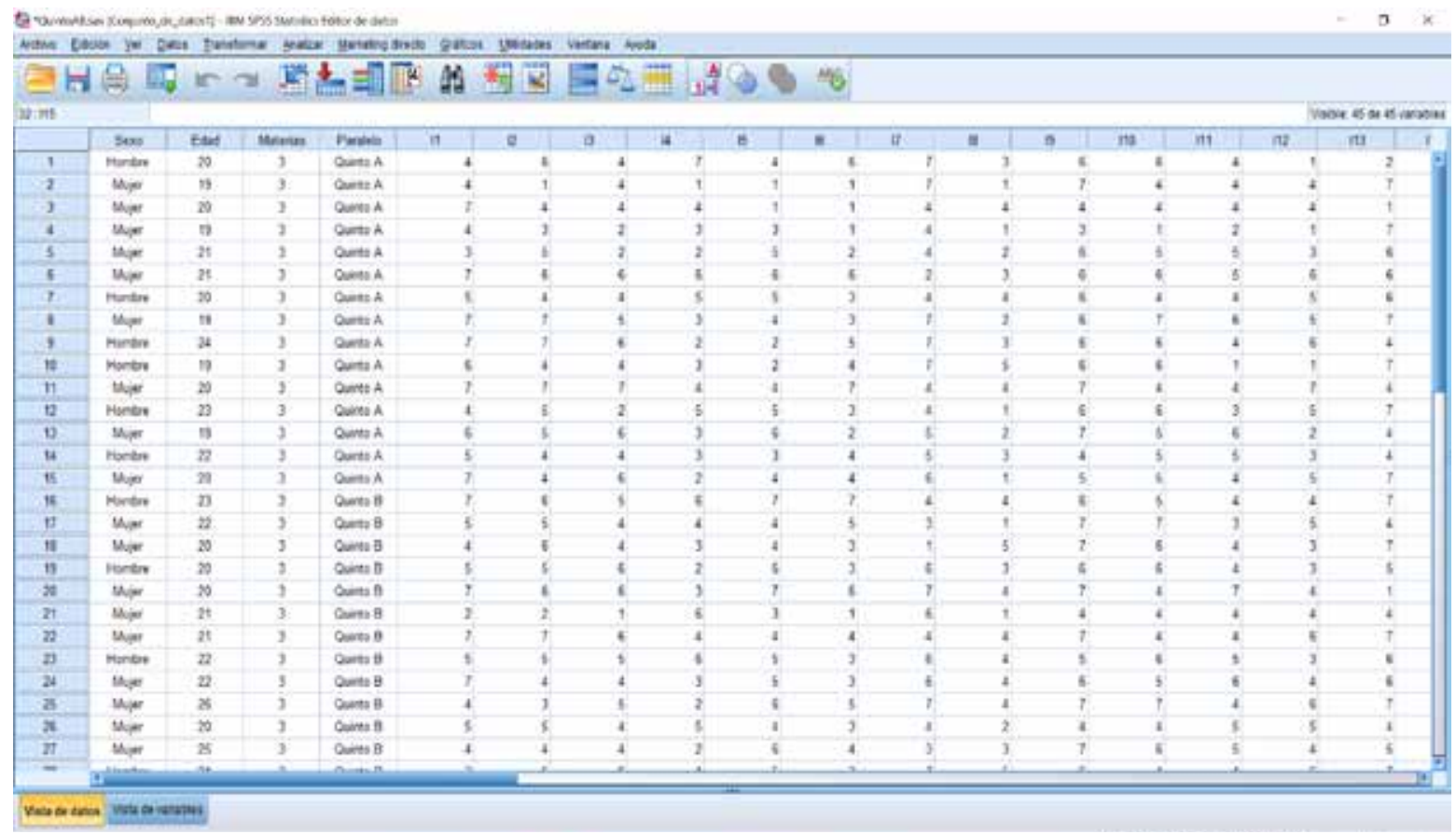

Nota: Extraído de las bitácoras de registro del equipo docente. 
Figura 9. Expresiones sobre el valor del Oráculo en las cápsulas audiovisuales
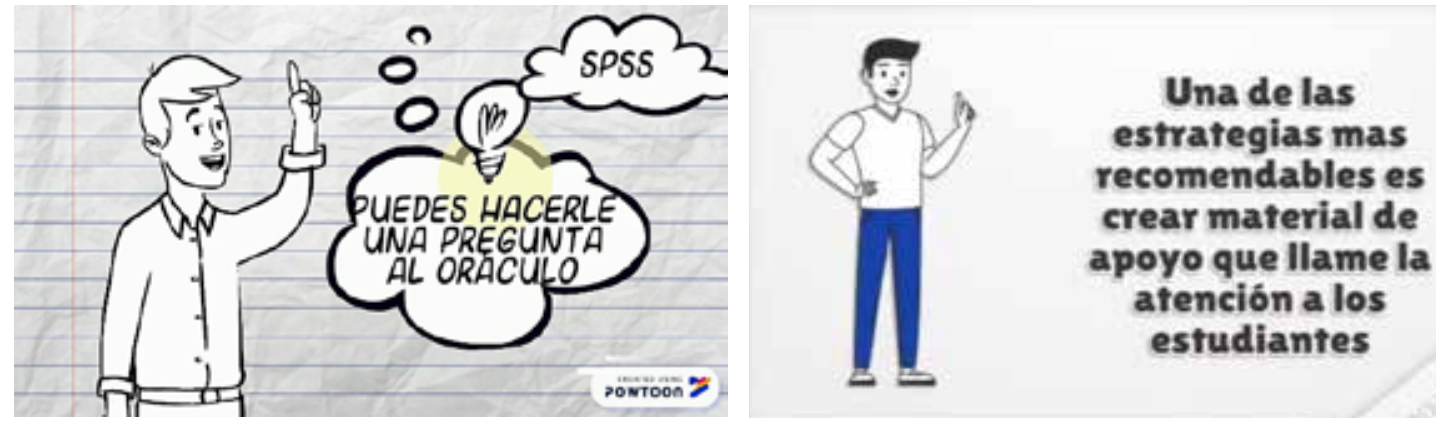

Nota: Extraídos de las cápsulas audiovisuales elaboradas por los estudiantes participantes.

Junto con el efecto anteriormente comentado, QuintoAB.sav cumplió una función social y educativa específica durante el módulo: romper el hielo (¡y el miedo!). Para cumplir con ello, la dinámica desplegada consistió en proyectar audiovisualmente la base de datos para luego invitar a los estudiantes a registrar en una hoja en blanco alguna pregunta que quisieran plantearle al Oráculo. Insistíamos en que, como nos habíamos asegurado de construir adecuadamente la fuente de información, solo bastaba con formular la pregunta correcta. Procuramos, a través de algunas instrucciones específicas, que los estudiantes formularan preguntas en un nivel descriptivo. Surgieron, de este modo, preguntas generales, como: ¿Qué edad tienen los estudiantes de quinto nivel? ¿Hay más mujeres $u$ hombres en nuestro grupo? ¿Tenemos una actitud positiva o negativa hacia la estadistica? Paulatinamente, fueron emergiendo preguntas de mayor especificidad: ¿Quiénes se interesan más por la estadistica: mujeres u hombres? ¿Quiénes valoran más la estadistica: el Paralelo A o el Paralelo B? ¿Quiénes tienen más dificultad con las estadisticas: las mujeres del Paralelo $A$ o las mujeres del Paralelo B? Esto se debió a las mediaciones realizadas por el equipo docente para orientar los cuestionamientos desde los aspectos más generales hacia los más específicos. Tomamos esta decisión, fundamentalmente, por nuestra necesidad de enseñar algunos elementos generales de estadística descriptiva (frecuencias y estadísticos de centro, dispersión y forma) y los respectivos procedimientos a ejecutar en el software utilizado en esta asignatura para describir datos cuantitativos (Menú-Comandos-Subcomandos, en el lenguaje de programación de IBM SPSS Statistics).

Junto con lo anterior, la función educativa de Quinto $A B$.sav se desplegó de la siguiente manera: primero registrábamos algunas de las preguntas formuladas por los estudiantes y luego las abríamos hacia el grupo curso. Por ejemplo, ¿Quiénes se interesan más por la estadistica: hombres o mujeres? El primer paso consistía en identificar el tipo de variable y su nivel de medición, lo cual era preguntado de forma abierta al grupo. En este ejemplo, consultábamos: ¿Qué tipo de variable es "Interés" y cuál es su nivel de medición? Esta pregunta nos permitía asegurar que los estudiantes comprendieran con exactitud qué tipo de análisis ejecutar si la variable era cualitativa o cuantitativa. Esta pregunta preliminar operaba según el principio de que el Oráculo necesita únicamente preguntas correctas para orientarnos de buena forma. En este ejemplo, tratándose de una variable cuantitativa con un nivel de medición escalar, procedíamos a ejecutar el procedimiento correspondiente en IBM SPSS Statistics: Menú Analizar- Comando Estadísticos Descriptivos - Subcomando Explorar, de modo tal que el Oráculo nos orientase precisamente respecto de las respuestas que buscábamos (Figura 10). 
PALACIOS-DÍAZ • CAISA-LOZADA • CAMACHO-JUMBO

Actitud hacia la estadística en estudiantes de Psicología: sistematización de una experiencia...

\section{Figura 10. El Oráculo en acción}

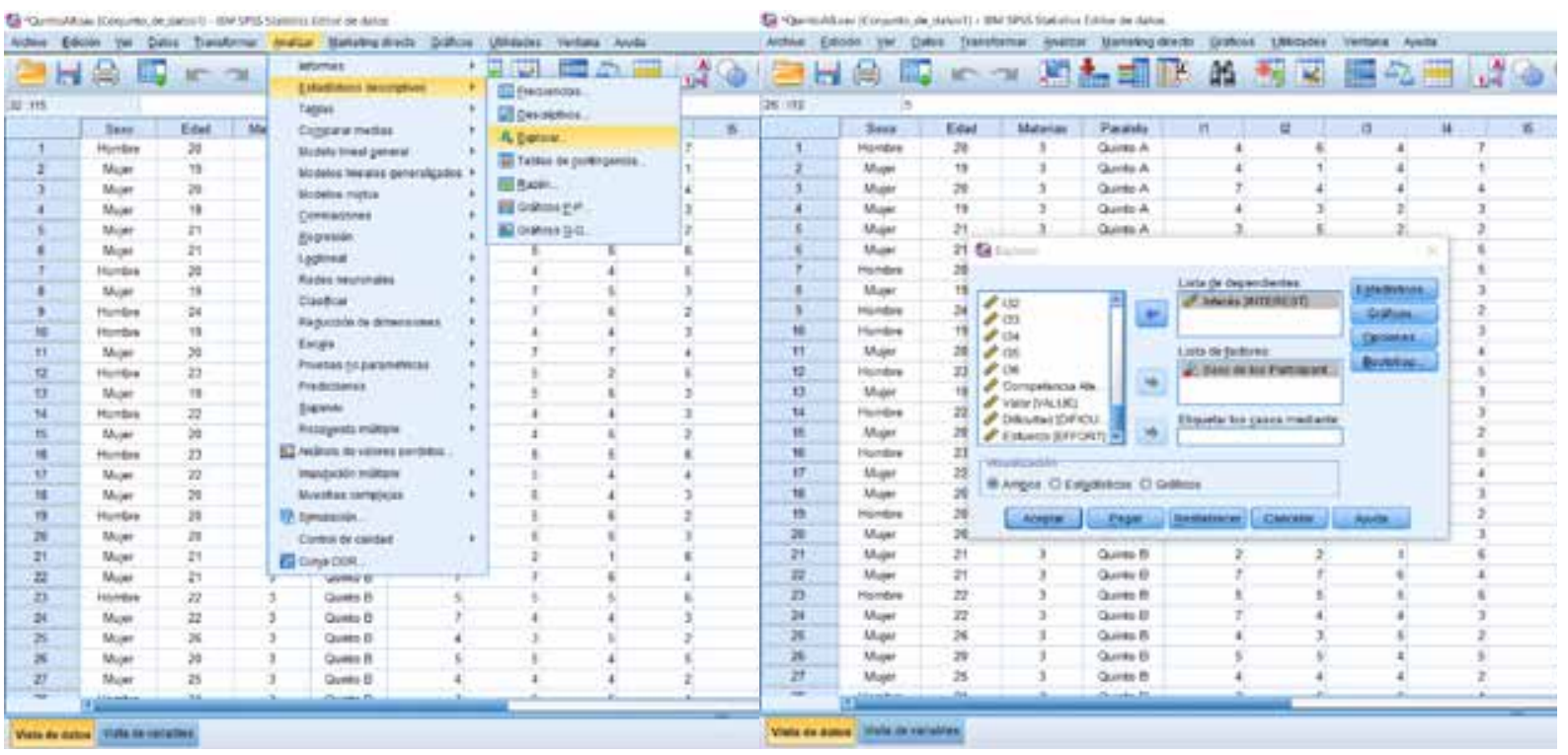

Nota: Extraído de las bitácoras de registro del equipo docente.

Tras este primer paso, orientábamos a los estudiantes a identificar la información realmente pertinente para las preguntas que nos habíamos formulado y a leerla correctamente desde un punto de vista estadístico. De esta manera, a partir del informe que arroja IBM SPSS Statistics, enseñábamos algunas formas de interpretar estadísticas de centro, dispersión y forma, así como también elementos gráficos. Una estrategia didáctica que resultó eficiente en esta experiencia fue lograr que los estudiantes reconocieran el carácter mentiroso de las medidas de centro. En ocasiones, solo observando las medias, los estudiantes se apresuraban en señalar que determinado perfil tenía mayor proporción de un atributo específico. Sin embargo, nos preocupamos de instalar una vigilancia epistemológica y metodológica en la lectura descriptiva de datos cuantitativos, para lo cual utilizábamos el breve texto del antipoeta chileno Nicanor Parra: "Hay dos panes. Usted se come dos, yo ninguno. Consumo promedio: un pan por persona”. Como las medidas de centro no son sensibles a determinadas variaciones, instábamos a los estudiantes a incorporar otros elementos (medidas de dispersión y forma, además de elementos gráficos) para examinar más cuidadosamente los datos y alcanzar así conclusiones más ajustadas.
Finalmente, los estudiantes habían desarrollado nociones básicas en gestión y análisis de bases de datos en IBM SPSS Statistics. Las sesiones 3 y 4 fueron un entrenamiento constante en los procedimientos anteriormente consignados. Por la misma razón, consideramos que era momento de que los estudiantes se planteasen a solas frente al Oráculo, pues ya contaban con herramientas no solo para formular correctamente preguntas sino también para, en un nivel descriptivo, navegar con precisión en el mar de posibilidades que se les ofrecía para obtener sus respuestas. Era un buen momento para embarcarse en la elaboración de un Informe de Análisis Estadistico y de demostrar y socializar lo aprendido.

\section{Socializando nuestra experiencia}

Hemos titulado Socializando nuestra experiencia al tercer momento de nuestra SE. Tras el trabajo de producción y análisis de datos realizado en las Sesiones 3 y 4 del Taller 2 de la asignatura, orientamos las actividades realizadas hacia la elaboración de dos productos: un Informe de Análisis Estadístico y una Cápsula Audiovisual para socializar los aprendizajes. Estos productos cumplieron una función social y educativa específica; en el primer caso, el Informe buscaba garantizar que los estudiantes habían incorporado 
fundamentos y procedimientos estadísticos básicos para analizar datos cuantitativos, mientras que en el segundo caso, la Cápsula Audiovisual pretendía que comunicaran e incentivaran a sus compañeros de niveles menores a comprometerse actitudinalmente con el aprendizaje de temas de estadística.

Para esta actividad final, se conformaron equipos de cinco personas y se entregó una guía titulada Actividad de Aprendizaje Práctico Experimental (Figura 11), que proponía como principal objetivo analizar descriptivamente los factores socioeducativos de los grupos y la actitud hacia la estadística de cada uno de ellos. De la misma manera, les solicitamos a los estudiantes que realizaran un análisis comparativo preliminar, lo cual fue finalmente el mayor alcance explicativo que logramos en el curso, incluso cuando habíamos definido preliminarmente un alcance relacional. Por último, les entregamos la consigna para la elaboración de la Cápsula Audiovisual con la cual socializarían sus aprendizajes a los estudiantes de niveles menores.

\section{Figura 11. Actividad de aprendizaje práctico experimental - Taller 2}

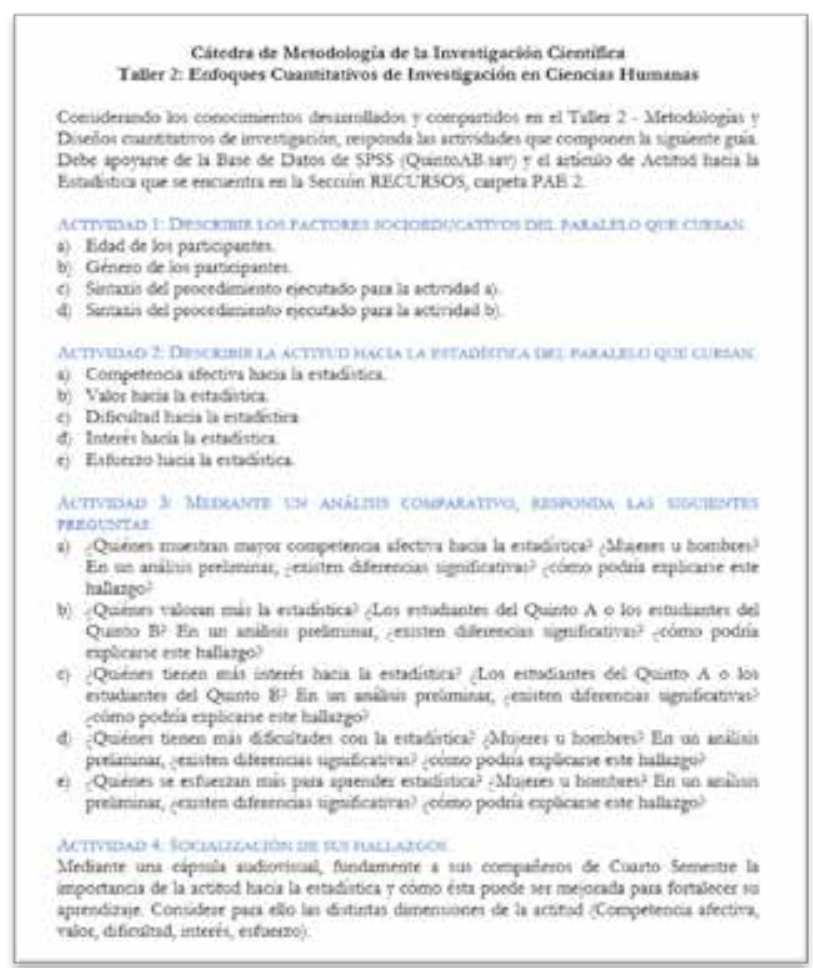

Nota: Extraído de las bitácoras de registro del equipo docente.
La elaboración de este informe estuvo guiada por un acompañamiento de dos tutorías académicas para cada grupo (una de carácter obligatorio y otra voluntaria). En ellas revisamos aspectos de redacción y ofrecimos algunas sugerencias técnicas para obtener mayor precisión en el análisis realizado. Del mismo modo, este fue un espacio abierto para complementar elementos que no habían sido suficientemente aprendidos en las distintas sesiones del curso. Finalmente, todos los grupos de estudiantes entregaron sus informes, obteniendo una calificación promedio de 8,5/10. Esta valoración numérica fue cotejada en conjunto con las Cápsulas Audiovisuales que los estudiantes elaboraron y, de modo más amplio, con entrevistas semiestructuradas realizadas cuatro semanas después de finalizado el Taller 2. Respecto a estas entrevistas, conviene indicar que fueron el producto central del Taller 3 - Enfoques Cualitativos de Investigación en Ciencias Humanas. En dicha instancia, los estudiantes debían construir un guion temático para evaluar la experiencia formativa de los talleres anteriores $y$, posteriormente, realizar una entrevista simulada a alguno de los participantes. Entregamos indicaciones técnicas y prácticas para que llevasen a cabo esta actividad, procurando que el guion temático estuviese coherentemente construido y que la interacción entre ellos mismos generase mayor fluidez en la expresión. De este modo, obtuvimos algunos elementos valiosos que los estudiantes pusieron de relieve de su experiencia formativa en el Taller 2. Nuevamente, un elemento fue la necesidad de afrontar el miedo a las estadísticas. Juan, estudiante del paralelo B, señala que:

Conforme han pasado los semestres, los profesores nos han implementado ese modelo de que no le tengamos miedo a la estadística, de que aprendamos más, de que preguntemos lo que no sepamos, de que participemos más. La estadística es una materia que es compleja, pero es bonita, y también es muy funcional, no solo para nuestra profesión, sino también para entender mejor por qué pasan las cosas. Por eso deberíamos seguir con estos métodos de enseñanza [Juan, Paralelo B, Entrevista Semiestructurada]. 
En este caso, se aprecia una valoración positiva de los métodos de enseñanza basados en la necesidad de perder el miedo a estos temas, lo cual está conectado con la apreciación tanto estética (es bonita) como práctica (es muy funcional), y el valor que esta materia puede tener para la vida cotidiana y el desempeño profesional. Un elemento adicional es destacado por Marta, estudiante del Paralelo A, quien resalta el papel de la confianza en la ardua tarea de perder el miedo a la estadística:

Es importante contar con confianza para asumir nuestra relación con la estadística, nuestra afectividad con la estadística, porque nadie quiere saber nada de la estadística, nadie quiere saber nada de los números, y tener confianza con tus docentes de estas materias es un aspecto importante para perder el miedo [Marta, Paralelo A, Entrevista Semiestructurada].

La confianza es posicionada como un gesto afectivo en la relación que los estudiantes establecen con los docentes, más en un contexto en el que prima el desinterés hacia la materia (nadie quiere saber nada de la estadística ni de los números). Este elemento relacional se configura a partir de una autocrítica que los estudiantes comparten respecto de su interés hacia la estadística y que conceptualizan a modo de un contagio. Roberto, estudiante del paralelo B, así lo expresa:

A veces las personas no le ponen tanta atención a la estadística, y eso se va compartiendo con los demás. Si veo que alguien no pone atención, eso se va contagiando por todo el curso, y así todo el curso no pone atención, todo el curso no le pone ganas, todo el curso no pregunta cuando no sabe nada, siempre se queda con la duda. Y ahí mismo vienen los problemas de no saber qué hacer en las clases de estadística, porque necesitamos estar atentos, pedir ayuda al profesor cuando no sepamos, pedir tutorías cuando estemos complicados [Roberto, $\mathrm{Pa}$ ralelo B, Entrevista Semiestructurada].

Esta actitud colectiva muchas veces resulta perjudicial para el proceso de aprendizaje de los grupos. El carácter contagioso del desinterés y la desatención genera muchas de las dinámicas que limitan las posibilidades de enseñar y aprender estadísticas en mejores condiciones. Esta autocrítica también es planteada como una oportunidad y una invitación a los docentes. Nuevamente Roberto es quien añade, con contundencia, que los docentes tienen un papel relevante en transitar desde aspectos concretos que sostengan el interés y la atención de los estudiantes, así como también son ambos quienes deben poner su mejor disposición al servicio de la experiencia formativa.

Es necesario poner esfuerzo desde ambas partes, tanto docentes como estudiantes. Como generalmente nuestra actitud es negativa hacia la estadística, solemos no prestar atención a las clases y nos vamos retrasando en relación con el ritmo de los compañeros. Por eso la explicación de los docentes tiene que plantearse desde los elementos más concretos de la materia porque si no, nos perdemos, es muy fácil distraerse y eso requiere que ambas partes tengamos la mejor disposición y la máxima motivación [Roberto, Paralelo B, Entrevista Semiestructurada].

\section{Discusión y conclusiones}

Esta sistematización de experiencias nos permitió reconstruir e interpretar críticamente el proceso formativo vivido en el Taller 2 - Enfoques Cuantitativos de Investigación en Ciencias Humanas de la cátedra de Metodología de la Investigación Científica. La SE es un método que nos ha posibilitado obtener aprendizajes desde la práctica con miras a comprender y transformar las condiciones en las que participamos en distintos procesos y eventos de la vida social. Como tal, esta experiencia investigativa nos ha facilitado no solo comprender detalladamente los perfiles actitudinales hacia la estadística de nuestros estudiantes, sino también desarrollar acciones concretas para orientar positivamente sus aprendizajes a través de un módulo formativo en específico.

A partir de ello, podemos destacar algunos resultados y hallazgos que pueden ser de interés para la comunidad científica y para los formadores en estadística en educación superior. En primer lugar, las actitudes hacia la estadística que han mostrado ambos paralelos se encuentran en línea con resultados previamente reportados, en el sentido de que tiende a haber 
actitudes neutras, levemente positivas (Landa, 2015; Pérez et al., 2015; Valencia, 2017), específicamente en las subdimensiones interés, valor y esfuerzo, pero también actitudes marcadamente negativas (Comas et al., 2017; Ordóñez et al., 2019; Vilá \& Rubio, 2016), especialmente en las subdimensiones dificultad y competencia afectiva para abordar temas y problemas estadísticos.

En segundo lugar, y recogiendo las recomendaciones didácticas realizadas por los investigadores de actitud hacia la estadística en el contexto iberoamericano contemporáneo, pensamos que esta SE contribuye específicamente a fortalecer las consideraciones relacionadas no solo con la dimensión didáctica de la enseñanza de la estadística, sino también con los elementos socioemocionales y relacionales que configuran las experiencias educativas y que, de no ser atendidos, pueden limitar los esfuerzos instruccionales de los formadores en esta materia. Particularmente, el miedo que manifiestan los estudiantes hacia estos temas se presenta dualmente como un obstáculo y como una oportunidad. En el primer caso, limita la capacidad percibida por los estudiantes para comprometerse actitudinal e intelectualmente con sus procesos formativos en la estadística, mientras que en el segundo permite transparentar cuáles son las dificultades percibidas, las experiencias que formaron el temor y la definición de cuáles pueden ser los pasos necesarios y cuáles no en el nivel sociocognitivo de la formación. Considerar estos elementos en la planeación y ejecución de las propuestas formativas puede constituir una poderosa herramienta para tensionar y redirigir un temor que, en general, es construido institucional y culturalmente. Esto tiene una implicación directa para los formadores, pues si, por ejemplo, nuestros estudiantes no son capaces de comprender la utilidad que la estadística puede tener para sus vidas cotidianas y profesionales, significa que nuestro pensamiento y acción pedagógica no es sensible a las problemáticas concretas que ellos tienen y, por tanto, no transmite con relevancia y pertinencia la potencia de estas herramientas para su desenvolvimiento educativo y social.
En tercer lugar, y atendiendo al contexto microcurricular y relacional del aula que configura las experiencias formativas en estadística, pensamos que es indispensable generar un mayor equilibrio entre nuestros programas y planificaciones y las necesidades y dificultades concretas de nuestros estudiantes. En el caso de la experiencia aquí sistematizada, contábamos con un grupo altamente comprometido con su aprendizaje y otro que manifestó explícitamente su desinterés hacia las estadísticas. No obstante, ambos grupos debían llegar al final de los propósitos definidos en nuestro mapa formativo. En función de esto, proponemos que los formadores en estadística debemos desarrollar una empatía basal hacia las disposiciones actitudinales de nuestros estudiantes, la cual debe atender mínimamente a una verificación constante del nivel de progreso en los contenidos (sin temor a regresar), a una capacidad para plantear concretamente los usos y utilidades de la estadística $y$, sobre todo, a involucrar cognitiva y afectivamente a los estudiantes en las posibilidades que ofrece esta herramienta para comprender y transformar la realidad social en que vivimos.

En cuarto lugar, quisiéramos invitar a los formadores en estadística a involucrase en procesos de sistematización de sus experiencias formativas en estos temas. Consideramos que hay en esta tarea una enorme oportunidad para tensionar la imagen arquetípica de seriedad y escasa claridad con que nos describen nuestros estudiantes, así como también para comprender más profundamente nuestras prácticas y orientarlas hacia el desarrollo de procesos formativos que respondan pertinentemente a las necesidades y dificultades de nuestros estudiantes. Ello también posibilitaría abordar y mejorar algunas de las limitaciones que ha tenido nuestro trabajo investigativo, como ha sido el hecho de abordar lo que es solo un módulo dentro de un curso más amplio, la dificultad de generar datos más enriquecidos de cada uno de los paralelos (tanto por la cantidad de estudiantes como de actividades a desarrollar dentro del semestre académico), y aspectos logísticos que podrían haber mejorado la experiencia como, por ejemplo, una medida post de actitudes hacia la estadística o entrevistas preliminares para comprender con mayor 
profundidad la experiencia de temor que individual y colectivamente manifiestan los estudiantes.

Finalmente, tras esta experiencia de SE pensamos que hay un valor político y ético a defender en la buena enseñanza de la estadística. Si es esta la ciencia del Estado y, con ello, la herramienta más poderosa para gobernar, orientar, disciplinar e intervenir grupos humanos y la población en general, los formadores en estadística somos actores fundamentales para enseñar a leer aguda y críticamente cómo datos y números pueden guiar (o no) a la humanidad hacia mejores condiciones de vida. Tras una sólida formación en fundamentos metodológicos y procedimientos técnicos para producir y analizar datos, hay una oportunidad para formar profesionales que, con su capacidad para leer estadísticamente la realidad social, sean también capaces de orientar sensiblemente con estos saberes y prácticas hacia procesos individuales y colectivos de toma de decisiones basados en los principios de una responsabilidad que no puede únicamente reducirse a lo individual, sino que debe contar también con aperturas solidarias hacia las problemáticas que afronta hoy la humanidad como especie, el mundo natural en que vivimos y el futuro incierto que nos depara si no transformamos decididamente nuestras formas de organización de la vida y, concretamente, de nuestras experiencias de aprendizaje.

\section{Referencias}

Armas, M. (2020). Estudio de las actitudes hacia la estadistica en alumnos universitarios. [Tesis doctoral, Universidad Complutense de Madrid]. E-Prints Complutense. https://eprints.ucm.es/59443/

Auzmendi, E. (1992). Las actitudes hacia la matemática-estadística en las enseñanzas media y universitaria. Bilbao: Ediciones Mensajero.

Cazorla, I. M., da Silva, C. B., Vendramini, C. A., \& Brito, M. R. F. (setiembre, 1999). Adaptação e validaçáo de uma escala de atitudes em relação à estatística [Adaptación y validación de una escala de actitudes hacia la estadística]. Trabajo presentado en la Conferencia internacional: Experiências e expectativas do Ensino da Estatística (pp. 45-58). Florianópolis, Brasil.

Comas, C., Martins, J., Nascimento, M., \& Estrada, A. (2017). Estudio de las actitudes hacia la estadística en estudiantes de Psicología. Bolema, 31(57), 479496. https://doi.org/10.1590/1980-4415v31n57a23

Estrada, A. (2002). Análisis de las actitudes y conocimientos estadísticos elementales en la formación del profesorado. [Tesis doctoral, Universidad Autónoma de Barcelona]. Dipòsit Digital de Documents de la UAB. https://ddd.uab.cat/record/38525

Estrada, A., Batanero, C., \& Fortuny, J. M. (2003). Actitudes y estadística en profesores en formación y en ejercicio. En Actas del 27 Congreso Nacional de Estadística e Investigación Operativa. Lleida: Edicions de la Universitat de Lleida.

Freire, P. (2012). Pedagogía del oprimido. Madrid: Siglo XXI.

Freire, P. (2014). Pedagogía de la autonomía. Saberes necesarios para la práctica educativa. Buenos Aires: Siglo XXI.

García-Martínez, J., Fallas-Vargas, M., \& Romero-Hernández, A. (2015). Las actitudes hacia la estadística del estudiantado de orientación. Revista Electrónica Educare, 19(1), 25-41.

https://doi.org/10.15359/ree.19-1.2

Gil-Flores, J. (1999). Actitudes hacia la estadística. Incidencia de las variables sexo y formación previa. Revista Española de Pedagogía, 214, 567-590.

Jara, O. (2015). La sistematización de experiencias: práctica y teoria para otros mundos posibles. Santiago de Chile: Quimantú.

Landa, M. (2015). Actitud hacia la estadística por parte de los estudiantes de Medición Psicológica de la UNAH, 2015. Ciencia y Tecnología, 17, 68-78. https://doi.org/10.5377/rct.v0i17.2681

Ordóńez, X., Romero, S., \& Ruiz, C. (2019). Actitudes hacia la estadística en alumnos de educación: análisis de perfiles. Revista de Educación, 385(3), 173-200. https://doi.org/10.4438/1988-592X-RE-2019-385-421 
Palacios, D. (2020). Sílabo metodología de la investigación cientifica. https://r.issu.edu.do/l?1=686God

Pérez, L., Aparicio, A., Bazán, J., \& Abdounur, O. (2015). Actitudes hacia la estadística de estudiantes universitarios de Colombia. Educación Matemática, 27(3), 111-139.

Rodríguez-Santero, J., \& Gil-Flores, J. (2019). Actitudes hacia la estadística en estudiantes de Ciencias de la Educación. Propiedades psicométricas de la versión española del Survey of Attitudes Toward Statistics (SATS-36). Relieve, 25(1), 1-17. https://doi.org/10.7203/relieve.25.1.12676

Schau, C., Stevens, J., Dauphinee, T. L., \& Del Vecchio, A. (1995). The development and validation of the survey of attitudes toward statistics [El desarrollo y la validación de una encuesta de actitudes hacia las estadísticas]. Educational and Psychological Measurement, 55(5), 868-875. https://doi.org/10.1177/0013164495055005022

Tobón, S. (2017). Ejes esenciales de la sociedad del conocimiento y la socioformación. Mount Dora: Kresearch.

Valencia, D. (2017). Actitudes hacia la estadística en estudiantes de Psicología de una universidad privada de Lima. Acta Psicológica Peruana, 27(1), 104-117.

Vilá, R., \& Rubio, M. (2016). Actitudes hacia la estadística en el alumnado del grado de Pedagogía de la Universidad de Barcelona. Revista de Docencia Universitaria, 14(1), 131-149.

https://doi.org/10.4995/redu.2016.5766

\section{CÓMO CITAR:}

Palacios-Díaz, D., Caisa-Lozada, E., \& Camacho-Jumbo, M. (2021). Actitud hacia la estadística en estudiantes de Psicología: Sistematización de una experiencia formativa. RECIE. Revista Caribeña de Investigación Educativa, 5(1), 40-60. https://doi. org/10.32541/recie.2021.v5i1.pp40-60 